\title{
Hiperlipidemias e Fatores Dietéticos: Estudo Transversal Entre Nipo-Brasileiros
}

\begin{abstract}
RESUMO
Este estudo teve como objetivo avaliar a associação entre hiperlipidemia e dieta de nipo-brasileiros de Bauru, município do Estado de São Paulo. Foram obtidos de 1.330 indivíduos dados mediante questionários previamente testados (dados demográficos e dietéticos). Os exames físicos e laboratoriais incluíram a coleta (ou dosagem) antropométrica, de pressão arterial, de glicemia de jejum e de 2 horas, de perfil lipídico e de TSH e T4 livre. Utilizaram-se teste qui-quadrado e medida de odds ratio para avaliar associação entre as variáveis estudadas e presença de hiperlipidemia. Foi encontrado $81,5 \%$ de hiperlipidêmicos e acometeu, principalmente, indivíduos tabagistas, com excesso de peso, hipotireoidismo, hipertensão arterial e intolerância a glicose. Observaram-se, mediante análise bruta, relações entre hiperlipidemia e ácido graxo saturado, ácido oléico e fibras de grãos e cereais. Após o ajuste para as variáveis de controle, observaram-se associações entre hiperlipidemia e consumo de lípides (totais, ácido oléico, saturados e trans), fibras e álcool. Conclui-se que mudanças no estilo de vida, particularmente no hábito alimentar, podem levar a uma melhora do quadro hiperlipidêmico e que o consumo de lípides pode ser um dos principais fatores para o aumento da hiperlipidemia. Estudos prospectivos auxiliarão para testar essas hipóteses nos nipo-brasileiros de Bauru. (Arq Bras Endocrinol Metab 2007;51/4:547-558)
\end{abstract}

Descritores: Hiperlipidemia; Fatores de risco; Fatores dietéticos; Nipobrasileiros

\begin{abstract}
Hyperlipidemias and Dietary Patterns: Transversal Study of Japanese Brazilians.

This study aimed at evaluating the association between hyperlipidemia and dietary patterns of Japanese Brazilians with and without hypothyroidism from Bauru, city in the State of São Paulo. We evaluated 1,330 individuals by means of demographic and dietary measurement, whom were gotten through standardized questionnaires previously tested. Clinical examination and laboratory data were anthropometry, blood pressure, fasting and 2-h glucose load, lipid profile and TSH and free T4. The chi-square and the odds ratio were used to evaluate associations between hyperlipidemia with studied variables. The prevalence of hyperlipidemia was $81.5 \%$ and it was associated with smokers, overweight, hypothyroid, hypertensive and glucose intolerants. We observed, in crude analysis, relationships with the presence of hyperlipidemia and fat saturated, oleic acid and dietary fiber from grains and cereals. After adjusting for the control variables, we observed relationships between hyperlipidemia (hypercholesterolemia and hypertriglyceridemia) with habitual intake of total fat, oleic acid, saturated fat, trans fat, dietary fiber and alcohol. As a conclusion, changes in the style of life, particularly in the dietary habits, can improve lipidic profile and that lipids intake can be a risk factor for hyperlipidemia. Prospectives studies will help test the hypothesis in Japanese Brazilians from Bauru. (Arq Bras Endocrinol Metab 2007;51/4:547-558)
\end{abstract}

Keywords: Hyperlipidemia; Risk factors; Dietary patterns; Japanese Brazilians artigo original

\author{
MARSELLE R. BeVILACQUA \\ SUELY G.A. GIMENO \\ LUIZA K. MATSUMURA \\ SANDRA R.G. FERREIRA \\ JAPANESE BRAZILIANS DIABETES \\ STUDY GROUP
}

Departamento de Medicina Preventiva (MRB, SGAG \& SRGF)

e Disciplina de Endocrinologia,

Departamento de Medicina (LKM), Universidade Federal de São Paulo, São Paulo, SP. 
A S HIPERLIPIDEMIAS SÃO ALTERAÇÕES metabólicas que ocorrem quando níveis de lípides circulantes estão aumentados na corrente sanguínea. Podem ser classificadas em hipercolesterolemia, hipertrigliceridemia e hiperlipidemia mista (1).

A proeminência da hiperlipidemia como problema de saúde pública está na sua relação com as doenças cardiovasculares, principalmente o acidente vascular cerebral e a doença aterosclerótica coronariana - razões importantes de morte e incapacidade física em nosso meio, com repercussões importantes nos custos da assistência médica no Brasil (2).

As alterações metabólicas, genéticas e ambientais que predispõem a aterogênese incluem fatores de risco modificáveis (hipertensão arterial, diabetes, tabagismo, dislipidemias, acúmulo de gordura visceral e sedentarismo) e não modificáveis (idade, sexo e história familiar). Quanto maior o impacto dos fatores de risco em um mesmo indivíduo, maior será o risco cardiovascular (3).

Segundo especialistas do NCEP (4), as principais causas secundárias para o aumento do colesterol e triglicérides sanguíneos são o hábito alimentar excessivo, e a secundária seria a presença do hipotireoidismo. Dados da Sociedade Brasileira de Endocrinologia e Metabologia do Distrito Federal, em 2004, estimam que $90 \%$ dos indivíduos com hipotireoidismo têm aumento nos níveis de colesterol ou de triglicérides (5).

O mecanismo primário da hipercolesterolemia nos hipotireóideos se dá pelo acúmulo de LDL, devido ao decréscimo do número correspondente de receptores hepáticos para a LDL. A reposição hormonal com tiroxina tem respostas variáveis sobre a hipercolesterolemia secundária, devido aos diferentes polimorfismos do gene do receptor de LDL. A redução da atividade da lipase das lipoproteínas, que pode ocorrer no hipotireoidismo, é a responsável pelo desenvolvimento de hipertrigliceridemia (3).

Sabe-se que, para uma melhor qualidade de vida, a dieta é um fator relevante. Indubitavelmente, não existe nenhuma prática ou hábito que possa influenciar tanto a saúde do indivíduo como as decisões que são tomadas com vistas ao tipo e quantidade de alimentos consumidos. A obtenção de nutrientes essenciais ao organismo depende da adequada seleção de alimentos, garantindo, assim, o seu funcionamento (6).

A dieta tradicional japonesa constitui um exemplo de padrão alimentar caracterizado pelo baixo consumo de açúcares simples, gordura e proteína animal, e rica em açúcares complexos. Com a emigração japonesa, as modificações na dieta têm despertado grande interesse em se avaliar a importância do fator ambiental no papel da obesidade e doenças associadas (7-9).
Hoje está bem estabelecido na literatura que mudanças no estilo de vida dos indivíduos são decisivas na conduta de tratamento ou prevenção da hiperlipidemia; essas mudanças incluem, entre outros fatores, a mudança no comportamento alimentar. O presente estudo teve como objetivo analisar a possível relação entre hiperlipidemia e os componentes da dieta de nipo-brasileiros com e sem hipotireoidismo que vivem em Bauru, SP, Brasil.

\section{MATERIAL E MÉTODOS}

Neste estudo, aprovado pelo Comitê de Ética da Universidade Federal de São Paulo - UNIFESP, utilizou-se dados de investigação epidemiológica do tipo transversal referentes à segunda fase de pesquisa realizada pelo Japanese Brazilians Diabetes Study Group com a comunidade nipo-brasileira de Bauru, cidade do interior do Estado de São Paulo, Brasil, no período de 1999 a 2000. Detalhes sobre as características, critérios de seleção e recrutamento dos nipo-brasileiros foram descritos anteriormente (9-12). Resumidamente, após censo da comunidade nipo-brasileira residente em Bauru, foram identificados e convidados a participar do estudo 1.751 indivíduos de primeira geração (nascidos no Japão) e segunda geração (nascidos no Brasil e filhos de indivíduos de primeira geração) com idade $\geq 30$ anos. O convite foi aceito por 1.330 $(76 \%)$ indivíduos. Os motivos para a não participação foram óbito (antes ou durante o trabalho de campo, $n=94 ; 22,3 \%$ ), mudança de endereço $(n=57 ; 13,5 \%)$ e recusa $(n=270$; $64,2 \%)$. Entre aqueles que não participaram do estudo, observou-se maior proporção de indivíduos do sexo masculino e com idade $\leq 60$ anos quando comparados aos demais participantes. Entre esses indivíduos não examinados, havia maior proporção de homens quando comparados às mulheres.

Após concordância em participar do estudo, formalizada mediante assinatura em termo de consentimento livre e esclarecido, os participantes foram entrevistados por pessoas treinadas utilizando questionários padronizados e previamente testados, a fim de obter informações relativas aos aspectos de identificação, características sócio-demográficas [sexo, idade, geração, tabagismo (nunca fumou, fuma atualmente ou já fumou alguma vez), prática de atividade física no lazer e no trabalho, histórico pessoal e familiar de doenças (hipertensão arterial sistêmica e intolerância à glicose)].

A atividade física foi avaliada por um questionário elaborado com o objetivo de colher informações sobre o tipo de atividade física executada tanto por lazer quanto no trabalho. No presente trabalho foram consideradas duas categorias de atividade física: leve e outros.

O consumo alimentar foi avaliado mediante a utilização de um questionário de freqüência do consumo alimentar, previamente validado para nipo-brasileiros (13). Os participantes foram questionados sobre o consumo habitual de alimentos e grupo de alimentos (122 itens) durante o ano anterior. Estes relataram a freqüência habitual de consumo 
de cada item, a unidade de tempo respectiva (se diário, semanal, mensal ou anual) e o tamanho da parcela habitual (se pequeno, médio, grande ou extra-grande com relação à parcela de referência para cada item do questionário). Utilizou-se o programa Dietsys 4.01 para o cálculo dos nutrientes da dieta (14). As bases de dados de nutrientes utilizadas foram as do Departamento de Agricultura dos Estados Unidos da América, além de uma tabela de composição de alimentos do Brasil (15) e do Japão (16). Com o objetivo de estudar a relação entre hiperlipidemia e variáveis dietéticas e a influência em indivíduos com e sem hipotireoidismo, foram analisados dados referentes ao consumo energético total, carboidratos, proteínas, lípides totais, ácido graxo linoléico, ácido graxo oléico, ácido graxo saturado, ácido graxo trans e colesterol, álcool, fibras totais e fibras em vegetais, grãos e cereais e frutas. Todos os nutrientes foram ajustados às calorias totais pelo método do resíduo (17) e agrupados em tercis de consumo.

Os exames físicos e laboratoriais foram realizados no Hospital de Reabilitação de Anomalias do Crânio-faciais em Bauru, SP. O peso corporal foi avaliado, em quilogramas, em balança antropométrica (Filizola ${ }^{\circledR}$ ) do tipo plataforma com capacidade de $200 \mathrm{~kg}$ e precisão $100 \mathrm{~g}$, colocada sob superfície plana e calibrada a cada pesagem. O indivíduo usava o mínimo de roupa possível e estava sem sapatos. A estatura foi tomada com o uso do estadiômetro manual, preso a parede com capacidade de dois metros e precisão de $1 \mathrm{~cm}$, estando o indivíduo descalço, com glúteos, tronco e cabeça de encontro à parede, sem rodapé e em superfície plana e lisa e com os braços pendentes ao longo do corpo. O índice de massa corporal (IMC) foi calculado com o peso, em quilogramas, dividido pela estatura ao quadrado, em metros. Utilizou-se as recomendações da WHO (18) para a classificação dos indivíduos quanto ao estado nutricional. Foram considerados eutróficos aqueles com IMC entre 18,5 a 24,9 $\mathrm{kg} / \mathrm{m}^{2}$, com sobrepeso, os com IMC entre 25 a $29,9 \mathrm{~kg} / \mathrm{m}^{2}$ e os obesos, os que apresentarem IMC $\geq 30 \mathrm{~kg} / \mathrm{m}^{2}$.

A circunferência de cintura foi medida com fita métrica inextensível, na altura da cicatriz umbilical, com aproximação em $0,1 \mathrm{~cm}$. Essa medida foi realizada com o indivíduo ereto, abdômen relaxado, braços ao lado do corpo e os pés juntos. A presença de obesidade central (ou abdominal) foi caracterizada por valores de circunferência de cintura $\geq 80$ e $90 \mathrm{~cm}$ para o sexo feminino e masculino, respectivamente (19).

As medidas de pressão arterial sistólica (PAS) e diastólica (PAD) foram realizadas por médicos treinados, utilizando-se aparelhos digitais automáticos com adequação do manguito à circunferência braquial, modelo HEM712C do fabricante $\mathrm{Omron}^{\circledR}$, após 10 minutos de repouso na posição sentada. Três medidas foram realizadas e o valor final considerado foi aquele que representou a média aritmética das duas últimas e foi expresso em milímetros de mercúrio $(\mathrm{mmHg})$. O critério para diagnóstico de hipertensão arterial foi baseado no sétimo relatório do comitê Norte-Americano de detecção, avaliação e tratamento de hipertensão arterial (20). Foram considerados hipertensos aqueles indivíduos que apresentaram valor de $\mathrm{PAS} \geq 140 \mathrm{mmHg}$ e $\mathrm{PAD} \geq 90$ $\mathrm{mmHg}$ ou em uso regular de medicação anti-hipertensiva.
Amostras de sangue foram coletadas após jejum de no mínimo 10 horas e após duas horas de ingestão de 75 gramas de glicose. Estas foram utilizadas para várias determinações incluindo o perfil lipídico, de glicose, de insulina e de função tireoideana. A sobrecarga com glicose foi feita para os indivíduos que não referiram diagnóstico anterior de DM e para aqueles que sabiam ser diabéticos, porém com glicemia de jejum capilar $<200 \mathrm{mg} / \mathrm{dL}$, medida por meio de glicosímetro (Glucostix/Glucometer System ${ }^{\circledR}$ ). A classificação dos indivíduos quanto ao grau de intolerância à glicose foi realizada segundo recomendação da WHO (21). Foram considerados normais os indivíduos com glicemia de jejum e de 2 horas < $110 \mathrm{mg} / \mathrm{dL}$ e $140 \mathrm{mg} / \mathrm{dL}$, respectivamente, com glicemia de jejum alterada (GJA), os indivíduos com glicemia de jejum < $110 \mathrm{mg} / \mathrm{dL}$ e glicemia de 2 horas entre 140-199 mg/dL, e com diabetes aqueles glicemia de jejum $\geq 126 \mathrm{mg} / \mathrm{dL}$ ou glicemia de jejum $\geq 200 \mathrm{mg} / \mathrm{dL}$ ou em uso de medicação antidiabética. A glicose plasmática foi determinada pelo método da glicose-oxidase.

Os lípides séricos foram medidos utilizando métodos enzimáticos. Os critérios de classificação obedeceram às normas do NCEP (4), onde foi classificado como hipertrigliceridemia o indivíduo com valor de triglicérides > 150 $\mathrm{mg} / \mathrm{dL}$ e com hipercolesterolemia aquele com colesterol total $>200 \mathrm{mg} / \mathrm{dL}$.

A dosagem dos hormônios tireoideanos (TSH e T4l) foi feita por meio de ensaio imunofluorimétrico ultra-sensível. Os valores considerados normais de referência para TSH e T4l foram, respectivamente, de $0,3-4,5 \mathrm{mU} / \mathrm{L}$ e 0,6-1,5 ng/dL (Wallac - Delphia, Finland). Foram considerados com hipotireoidismo clínico aqueles com TSH > 4,5 $\mathrm{mU} / \mathrm{L}$ e $\mathrm{T} 4 \mathrm{~L}<0,6 \mathrm{ng} / \mathrm{dL}$, e hipotireoidismo subclínico aqueles com TSH entre 0,3 a $4,5 \mathrm{mU} / \mathrm{L}$ e $\mathrm{T} 4 \mathrm{~L}<0,6$ $\mathrm{ng} / \mathrm{dL}$. Neste estudo, ambos os grupos (clínico e subclínico) foram considerados como com hipotireoidismo.

\section{Análise dos dados}

As comparações das freqüências de indivíduos com ou sem hiperlipidemia segundo variáveis de saúde, sócio-demográficas e antropométricas foram feitas mediante a utilização do teste quiquadrado. Utilizou-se, como medida de associação, o odds ratio (OR) por ponto e por intervalo com 95\% de confiança (IC95\%).

De forma a descrever os dados, os valores das variáveis dietéticas foram agrupados em tercis de consumo e, para cada tercil, foram obtidos os valores médios e do desviopadrão do consumo do nutriente, o número de casos de hiperlipidemia e os valores dos OR.

Os efeitos das variáveis de principal interesse calorias totais, carboidratos, lípides, proteínas, ácido graxo saturado, ácido oléico, ácido linoléico, ácido graxo trans, álcool, fibras totais, fibras de vegetais, fibras de cereais, fibras de frutas - sobre a hiperlipidemia foram ajustados para variáveis de controle (sexo, idade, hábito de fumar, estado nutricional, obesidade central, diabetes mellitus, hipertensão arterial) com a utilização do modelo de regressão logística. Essas variáveis foram selecionadas por apresentarem, na análise bruta, valor de $\mathrm{p}<0,20$. Dada a importância da re- 
lação entre as hiperlipidemias e o hipotireoidismo e, uma vez que o número de indivíduos com essa doença tireoideana $(\mathrm{n}=121)$ não permitiu a análise entre aqueles com e sem essa condição, optou-se por fazê-lo apenas entre os sem hipotireoidismo, retirando-se como conseqüência essa variável do modelo de regressão logística. Esses procedimentos foram repetidos considerando, como variável resposta, tanto a hipercolesterolemia quanto a hipertrigliceridemia, isoladamente.

Os dados foram analisados com a utilização do programa estatístico STATA (versão 8.2 para Windows) para microcomputador (22).

\section{RESULTADOS}

Entre os 1.330 indivíduos que participaram desse estudo, $81,5 \%(\mathrm{n}=1.084)$ apresentaram algum tipo de hiperlipidemia, sendo que a maior proporção foi en- contrada nos homens ( $83,7 \%$ vs. $79,6 \%)$ e indivíduos com mais de 60 anos $(84,8 \%$ vs. $79,2 \%)$, quando comparados, respectivamente, as mulheres e aqueles com idade $<60$ anos. As porcentagens de indivíduos com hipertrigliceridemia ou hipercolesterolemia isoladas foram, respectivamente, $65,6 \%$ e $62 \%$. Na tabela 1 apresentam-se as características demográficas, antropométricas e de saúde dos nipo-brasileiros segundo a presença ou ausência de hiperlipidemia. Observa-se que indivíduos hiperlipidêmicos quando comparados aos normais são, em geral, mais velhos $(\mathrm{OR}=1,47$; IC95\%= $1,10-1,97)$, tabagistas $(\mathrm{OR}=1,77$; IC95\%= 1,27-2,45), apresentam mais freqüentemente obesidade abdominal $(\mathrm{OR}=3,24 ; \mathrm{IC} 95 \%=2,34-4,49)$, sobrepeso $(\mathrm{OR}=3,07$; $\mathrm{IC} 95 \%=2,18-4,33)$, obesidade $(\mathrm{OR}=3,07$; IC95\%= 1,69-5,57), hipertensão $(\mathrm{OR}=2,45 ; \quad \mathrm{IC} 95 \%=$ 1,80-3,32), intolerância à glicose (glicose de jejum

Tabela 1. Número e porcentagem de nipo-brasileiros segundo presença de hiperlipidemia e variáveis demográficas, antropométricas e de saúde.

\begin{tabular}{|c|c|c|c|c|c|c|c|c|c|c|}
\hline \multirow[t]{3}{*}{ Variável } & & \multicolumn{4}{|c|}{ Hiperlipidemia } & & & \multirow[t]{3}{*}{$\chi^{2}(\mathbf{p})$} & \multirow[t]{3}{*}{ OR } & \multirow[t]{3}{*}{ IC95\% } \\
\hline & & \multicolumn{2}{|c|}{ Sim } & \multicolumn{2}{|c|}{ Não } & \multicolumn{2}{|c|}{ Total } & & & \\
\hline & & $\mathbf{N}$ & $\%$ & $\mathbf{N}$ & $\%$ & $\mathbf{N}$ & $\%$ & & & \\
\hline \multirow[t]{2}{*}{ Sexo } & Masculino & 514 & 83,7 & 100 & 16,3 & 614 & 100 & $3,69(0,06)$ & 1,32 & $0,99-1,74$ \\
\hline & Feminino & 570 & 79,6 & 146 & 20,4 & 716 & 100 & & & \\
\hline \multirow[t]{2}{*}{ Geração } & Segunda & 868 & 81,1 & 203 & 18,9 & 1071 & 100 & $0,77(0,38)$ & 0,85 & $0,59-1,22$ \\
\hline & Primeira & 216 & 83,4 & 43 & 16,6 & 259 & 100 & & & \\
\hline \multirow[t]{2}{*}{ Idade } & $>60$ anos & 459 & 84,8 & 82 & 15,2 & 541 & 100 & $6,74(0,009)$ & 1,47 & $1,10-1,97$ \\
\hline & $\leq 60$ anos & 625 & 79,2 & 164 & 20,8 & 789 & 100 & & & \\
\hline \multirow{2}{*}{$\begin{array}{l}\text { Atividade física } \\
\text { no trabalho }\end{array}$} & Leve & 265 & 79,1 & 70 & 20,9 & 335 & 100 & $0,002(0,96)$ & 1,01 & $0,69-1,47$ \\
\hline & Outros & 248 & 79,0 & 66 & 21,0 & 314 & 100 & & & \\
\hline \multirow{2}{*}{$\begin{array}{l}\text { Atividade física } \\
\text { no lazer }\end{array}$} & Leve & 723 & 81,2 & 167 & 18,8 & 890 & 100 & $0,07(0,79)$ & 1,04 & $0,77-1,40$ \\
\hline & Outros & 347 & 81,8 & 77 & 18,2 & 424 & 100 & & & \\
\hline \multirow[t]{2}{*}{ Tabagismo } & $\operatorname{Sim}^{1}$ & 359 & 86,9 & 54 & 13,1 & 413 & 100 & $11,79(0,001)$ & 1,77 & $1,27-2,45$ \\
\hline & Não & 719 & 79,0 & 191 & 21,0 & 910 & 100 & & & \\
\hline Obesidade & $\operatorname{Sim}^{2}$ & 510 & 90,6 & 53 & 9,4 & 563 & 100 & $53,58(0,000)$ & 3,24 & $2,34-4,49$ \\
\hline Central & Não & 573 & 74,8 & 193 & 25,2 & 766 & 100 & & & \\
\hline \multirow{3}{*}{$\begin{array}{l}\text { Estado } \\
\text { nutricional3 }\end{array}$} & Normal & 538 & 74,4 & 185 & 25,6 & 723 & 100 & $56,66(0,000)$ & 1 & \\
\hline & Sobrepeso & 429 & 89,9 & 48 & 10,1 & 477 & 100 & & 3,07 & $2,18-4,33$ \\
\hline & Obeso & 116 & 89,9 & 13 & 10,1 & 129 & 100 & & 3,07 & $1,69-5,57$ \\
\hline \multirow[t]{2}{*}{ Hipotireoidismo } & Sim & 108 & 89,3 & 13 & 10,7 & 121 & 100 & $5,24(0,02)$ & 1,98 & $1,09-3,59$ \\
\hline & Não & 864 & 80,7 & 206 & 19,3 & 1070 & 100 & & & \\
\hline Hipertensão & Sim & 518 & 88,6 & 67 & 11,4 & 585 & 100 & $34,5(0,000)$ & 2,45 & $1,80-3,32$ \\
\hline Arterial & Não & 565 & 75,9 & 179 & 24,1 & 744 & 100 & & & \\
\hline Tolerância & Normal & 40 & 58,0 & 29 & 42,0 & 69 & 100 & $64,39(0,000)$ & 1 & \\
\hline \multirow[t]{3}{*}{ a glicose 4} & GJA & 349 & 74,6 & 119 & 25,4 & 468 & 100 & & 2,13 & $1,26-3,58$ \\
\hline & TGD & 263 & 84,6 & 48 & 15,4 & 311 & 100 & & 3,97 & $2,25-7,01$ \\
\hline & $\mathrm{DM}$ & 432 & 89,8 & 49 & 10,2 & 481 & 100 & & 6,39 & $3,64-11,21$ \\
\hline
\end{tabular}

\footnotetext{
1 Hábito de fumar (sim: atual/passado e não: nunca)

2 Circunferência de cintura (sim: homens $\geq 90 \mathrm{~cm}$ e mulheres $\geq 80 \mathrm{~cm}$ e não: homens $<90 \mathrm{~cm}$ e mulheres $<80 \mathrm{~cm}$ )

3 IMC: Normal< 24,99 kg/m²; Sobrepeso: 25,0 a 29,99 kg/m² e Obesidade: > $30 \mathrm{~kg} / \mathrm{m}^{2}$

4 GJA - Glicemia de jejum alterada; TGD - Tolerância à glicose diminuída; DM - Diabetes mellitus
} 
alterada $\mathrm{OR}=2,13, \mathrm{IC} 95 \%=1,26-3,58)$, tolerância à glicose diminuída $(\mathrm{OR}=3,97, \mathrm{IC} 95 \%=2,25-7,01)$ e diabetes $(\mathrm{OR}=6,39, \mathrm{IC} 95 \%=3,64-11,21)$. Ainda na tabela 1 , observa-se maior prevalência de hiperlipidemia entre os indivíduos com hipotireoidismo $(\mathrm{OR}=1,98$; IC95\%= 1,09-3,59) quando comparados aos sem essa condição. Destaca-se que, ao considerar, separadamente, as relações entre hipotireoidismo e hipercolesterolemia $(\mathrm{OR}=1,15 ; \mathrm{IC} 95 \%=0,77-1,76)$ ou hipertrigliceridemia $(\mathrm{OR}=1,53 ; \mathrm{IC} 95 \%=0,99-2,42)$, estas não foram estatisticamente significantes.

Na tabela 2 estão apresentados, para cada tercil de consumo dos diferentes nutrientes, os valores médios das variáveis dietéticas, a prevalência de

Tabela 2. Média (desvio-padrão) e número (porcentagem) de nipo-brasileiros com hiperlipidemia e valores dos odds ratios segundo tercil das variáveis dietéticas.

\begin{tabular}{|c|c|c|c|c|}
\hline \multirow[t]{2}{*}{ Variável } & & \multicolumn{3}{|c|}{ Tercis de nutriente } \\
\hline & & $1^{\circ}$ tercil & $2^{\circ}$ tercil & $3^{\circ}$ tercil \\
\hline \multirow[t]{3}{*}{ Calorias totais (kcal) } & Média (dp) & $1379,2(224,7)$ & $1918,0(139,1)$ & $2670,8(518,8)$ \\
\hline & $\mathrm{N}^{\circ}(\%)$ casos & $343(80,3)$ & $348(81,3)$ & $353(82,5)$ \\
\hline & OR (IC95\%) & 1 & $1,07(0,76-1,50)$ & $1,15(0,82-1,62)$ \\
\hline \multirow[t]{3}{*}{ Carboidratos (g) } & Média (dp) & $226,0(103,5)$ & $263,2(74,0)$ & $306,1(65,9)$ \\
\hline & $\mathrm{N}^{\circ}(\%)$ casos & $332(80,2)$ & $351(80,0)$ & $361(84,2)$ \\
\hline & OR (IC95\%)1 & 1 & $0,97(0,70-1,36)$ & $1,31(0,92-1,87)$ \\
\hline \multirow{3}{*}{ Proteínas (g) } & Média (dp) & $54,6(23,4)$ & $67,8(20,7)$ & $81,8(22,4)$ \\
\hline & $\mathrm{N}^{\circ}(\%)$ casos & $355(82,6)$ & $340(82,1)$ & $349(79,5)$ \\
\hline & OR (IC95\%) ${ }^{1}$ & 1 & $0,97(0,68-1,38)$ & $0,82(0,58-1,15)$ \\
\hline \multirow[t]{3}{*}{ Lípides totais (g) } & Média (dp) & $58,8(28,7)$ & $72,3(26,2)$ & $85,7(20,7)$ \\
\hline & $\mathrm{N}^{\circ}(\%)$ casos & $365(83,9)$ & $336(79,8)$ & $343(80,3)$ \\
\hline & OR (IC95\%) ${ }^{1}$ & 1 & $0,76(0,53-1,08)$ & $0,78(0,55-1,11)$ \\
\hline \multirow[t]{3}{*}{ Colesterol (mg) } & Média (dp) & $118,9(70,8)$ & $180,2(66,8)$ & $267,7(113,5)$ \\
\hline & $\mathrm{N}^{\circ}(\%)$ casos & $360(83,7)$ & $343(82,1)$ & $341(78,4)$ \\
\hline & OR (IC95\%) ${ }^{1}$ & 1 & $0,89(0,62-1,27)$ & $0,70(0,50-1,00)$ \\
\hline \multirow[t]{3}{*}{ Ácido graxo Saturado (g) } & Média (dp) & $12,7(7,0)$ & $17,3(6,9)$ & $21,8(6,2)$ \\
\hline & $\mathrm{N}^{\circ}(\%)$ casos & $372(85,9)$ & $348(81,7)$ & $324(76,4)$ \\
\hline & OR $(\text { IC95\%) })^{1}$ & 1 & $0,73(0,51-1,05)$ & $0,53(0,37-0,76)$ \\
\hline \multirow[t]{3}{*}{ Ácido linoléico (g) } & Média (dp) & $8,7(4,3)$ & $11,2(3,2)$ & $14,9(4,9)$ \\
\hline & $\mathrm{N}^{\circ}(\%)$ casos & $355(83,3)$ & $348(81,9)$ & $341(78,9)$ \\
\hline & OR (IC95\%) ${ }^{1}$ & 1 & $0,90(0,63-1,29)$ & $0,75(0,53-1,06)$ \\
\hline \multirow[t]{3}{*}{ Ácido oléico (g) } & Média (dp) & $26,0(12,7)$ & $27,2(9,4)$ & $28,3(9,8)$ \\
\hline & $\mathrm{N}^{\circ}(\%)$ casos & $371(85,9)$ & $325(78,1)$ & $348(80,0)$ \\
\hline & OR (IC95\%) ${ }^{1}$ & 1 & $0,59(0,41-0,84)$ & $0,66(0,46-0,94)$ \\
\hline \multirow[t]{3}{*}{ Ácido graxo trans (g) } & Média (dp) & $6,3(1,8)$ & $11,0(1,5)$ & $21,7(12,2)$ \\
\hline & $\mathrm{N}^{\circ}(\%)$ casos & $325(78,5)$ & $341(83,2)$ & $345(83,3)$ \\
\hline & OR (IC95\%) ${ }^{1}$ & 1 & $1,36(0,95-1,92)$ & $1,37(0,97-1,94)$ \\
\hline \multirow[t]{3}{*}{ Álcool (g) } & Média (dp) & $0,007(0,02)$ & $0,01(0,05)$ & $15,2(23,6)$ \\
\hline & $\mathrm{N}^{\circ}(\%)$ casos & $341(80,8)$ & $328(77,4)$ & $375(85,8)$ \\
\hline & OR (IC95\%) ${ }^{1}$ & 1 & $0,81(0,58-1,13)$ & $1,4(1,00-2,06)$ \\
\hline \multirow[t]{3}{*}{ Fibras totais $(\mathrm{g})$} & Média (dp) & $22,8(8,1)$ & $11,9(5,4)$ & $16,8(6,3)$ \\
\hline & $\mathrm{N}^{\circ}(\%)$ casos & $382(83,0)$ & $280(81,9)$ & $382(79,4)$ \\
\hline & OR (IC95\%) ${ }^{1}$ & 1 & $0,92(0,64-1,33)$ & $0,79(0,57-1,09)$ \\
\hline \multirow[t]{3}{*}{ Fibras de cereais $(\mathrm{g})$} & Média (dp) & $2,1(1,0)$ & $3,2(1,0)$ & $4,8(1,8)$ \\
\hline & $\mathrm{N}^{\circ}(\%)$ casos & $351(83,8)$ & $356(82,0)$ & $337(78,4)$ \\
\hline & OR (IC95\%)1 & 1 & $0,88(0,62-1,26)$ & $0,70(0,50-0,99)$ \\
\hline \multirow[t]{3}{*}{ Fibras de vegetais $(\mathrm{g})$} & Média (dp) & $0,05(0,4)$ & $0,2(0,7)$ & $0,6(0,9)$ \\
\hline & $\mathrm{N}^{\circ}(\%)$ casos & $345(83,1)$ & $316(78,2)$ & $336(81,8)$ \\
\hline & OR (IC95\%) ${ }^{1}$ & 1 & $0,73(0,51-1,03)$ & $0,91(0,64-1,30)$ \\
\hline \multirow[t]{3}{*}{ Fibras das frutas $(\mathrm{g})$} & Média (dp) & $4,8(2,2)$ & $8,8(2,6)$ & $15,1(6,0)$ \\
\hline & $\mathrm{N}^{\circ}(\%)$ casos & $344(80,9)$ & $348(80,7)$ & $352(82,4)$ \\
\hline & OR (IC95\%) 1 & 1 & $0,99(0,70-1,38)$ & $1,11(0,78-1,56)$ \\
\hline
\end{tabular}

1 Valores ajustados às calorias totais 
hiperlipidemia e os valores de OR. Observou-se menor prevalência de hiperlipidemia entre os indivíduos com maior consumo de ácido oléico quando comparados aos com menor ingestão habitual desse nutriente $\left(3^{\circ}\right.$ vs. $1^{\circ}$ tercil: $\mathrm{OR}=0,66 ; \mathrm{IC} 95 \%=0,46-0,94 ; 2^{\circ}$ vs. $1^{\circ}$ tercil: $\mathrm{OR}=0,59 ; \mathrm{IC} 95 \%=0,41-0,84)$. Situação semelhante foi também observada entre aqueles com maior consumo de fibras provenientes de cereais $\left(3^{\circ}\right.$ vs. $1^{\circ}$ tercil: $\mathrm{OR}=0,70 ; \mathrm{IC} 95 \%=0,50-0,99)$. Diferentemente do esperado, observou-se que entre aqueles com maior consumo de ácidos graxos saturados, a prevalência de hiperlipidemia foi menor que a observada entre os que relataram menor consumo habitual desse nutriente $\left(3^{\circ}\right.$ vs. $1^{\circ}$ tercil: $\left.\mathrm{OR}=0,53 ; \mathrm{IC} 95 \%=0,37-0,76\right)$.

Os resultados obtidos com a utilização do modelo de regressão logística indicaram existência de associação entre hiperlipidemia e o consumo habitual de lípides totais, ácidos graxos saturados e ácidos graxos trans (tabela 3 ), independentemente do sexo, idade, hábito de fumar, estado nutricional, obesidade central, diabetes mellitus, hipertensão arterial e hipotireoidismo (lípides totais: $3^{\circ}$ vs. $1^{\circ}$ tercil: $\mathrm{OR}=$ 2,40; IC95\%= 1,11-5,23; ácidos graxos saturados: $3^{\circ}$ vs. $1^{\circ}$ tercil: $\mathrm{OR}=0,49 ; \mathrm{IC} 95 \%=0,26-0,91$; ácidos graxos trans: $3^{\circ}$ vs. $1^{\circ}$ tercil $\mathrm{OR}=1,65 ; \mathrm{IC} 95 \%=$ $1,10-2,47)$. Quando excluídos desse modelo os indivíduos com hipotireoidismo, além das associações an- teriormente encontradas, o consumo de carboidratos também foi significante $\left(3^{\circ}\right.$ vs. $1^{\circ}$ tercil: $O R=1,92$; IC95\% $=1,02-3,63$ ).

$\mathrm{Na}$ tabela 4 apresentam-se os valores dos OR ajustados, considerando, separadamente, como variável resposta a presença ou ausência de hipercolesterolemia ou hipertrigliceridemia. Observa-se que a presença de hipercolesterolemia associou-se, independentemente do sexo, idade, hábito de fumar, estado nutricional, obesidade central, diabetes mellitus, hipertensão arterial e hipotireoidismo, a ingestão habitual de lípides totais ( $3^{\circ}$ vs. $1^{\circ}$ tercil: $\mathrm{OR}=1,87$; IC $\left.95 \%=1,07-3,27\right)$, de fibras totais $\left(2^{\circ}\right.$ vs. $1^{\circ}$ tercil: $\mathrm{OR}=1,49$; IC $95 \%=$ 1,05-2,12) e de ácidos graxos trans $\left(3^{\circ}\right.$ vs. $1^{\circ}$ tercil: $\mathrm{OR}=1,39 ; \mathrm{IC} 95 \%=1,03-1,88)$. Quando excluídos do modelo os indivíduos com hipotireoidismo, apenas a associação para lípides totais perde a significância.

Ainda na tabela 4, observam-se associações entre a presença de hipertrigliceridemia com o consumo de álcool ( $3^{\circ}$ vs. $1^{\circ}$ tercil: $\mathrm{OR}=1,48$; IC95\%= 1,03-2,13) e de ácido oléico ( $2^{\circ}$ vs. $1^{\circ}$ tercil: $\mathrm{OR}=$ $0,70 ;$ IC95\% $=0,50-0,98)$, independentemente das variáveis de controle. Quando excluídos do modelo os indivíduos com hipotireoidismo, além de persistir a associação entre ácido oléico, o consumo de carboidrato associou-se a presença de hipertrigliceridemia $\left(3^{\circ}\right.$ vs. $1^{\circ}$ tercil: $\left.\mathrm{OR}=1,91 ; \mathrm{IC} 95 \%=1,12-3,25\right)$.

Tabela 3. Valores dos odds ratios ajustados (intervalo com $95 \%$ de confiança) obtidos para a presença de hiperlipidemia e tercis dos nutrientes.

\begin{tabular}{|c|c|c|c|}
\hline Variável1 & $\begin{array}{c}1^{\circ} \text { tercil } \\
\text { OR }(\text { IC } 95 \%)^{2}\end{array}$ & $\begin{array}{c}\text { Nutriente } \\
2^{\circ} \text { tercil } \\
\text { OR }(\text { (IC95\%)2 }\end{array}$ & $\begin{array}{c}3^{\circ} \text { tercil } \\
\text { OR (IC95\%)2 }\end{array}$ \\
\hline Calorias totais (kcal) & 1 & $0,99(0,581-1,67)$ & $1,01(0,561-1,81)$ \\
\hline Carboidratos (g) & 1 & $1,17(0,741-1,86)$ & $1,65(0,901-3,04)$ \\
\hline Proteínas (g) & 1 & $1,22(0,771-1,93)$ & $1,19(0,711-1,89)$ \\
\hline Lípides totais (g) & 1 & $1,23(0,701-2,14)$ & $2,41(1,111-5,23)$ \\
\hline Colesterol (mg) & 1 & $0,92(0,571-1,50)$ & $0,86(0,501-1,49)$ \\
\hline Ácido graxo saturado (g) & 1 & $0,68(0,411-1,11)$ & $0,49(0,261-0,91)$ \\
\hline Ácido linoléico (g) & 1 & $1,03(0,641-1,67)$ & $0,79(0,431-1,45)$ \\
\hline Ácido oléico (g) & 1 & $0,68(0,441-1,06)$ & $0,82(0,511-1,34)$ \\
\hline Ácido graxo trans (g) & 1 & $1,46(0,971-2,19)$ & $1,65(1,101-2,47)$ \\
\hline Álcool (g) & 1 & $0,90(0,531-1,54)$ & $1,53(0,961-2,44)$ \\
\hline Fibras totais $(\mathrm{g})$ & 1 & $1,36(0,841-2,21)$ & $0,87(0,581-1,30)$ \\
\hline Fibras de cereais $(\mathrm{g})$ & 1 & $0,98(0,631-1,52)$ & $0,82(0,521-1,30)$ \\
\hline Fibras de vegetais $(\mathrm{g})$ & 1 & $0,74(0,481-1,13)$ & $1,00(0,641-1,56)$ \\
\hline Fibras das frutas $(\mathrm{g})$ & 1 & $1,17(0,721-1,91)$ & $0,85(0,471-1,55)$ \\
\hline
\end{tabular}


Tabela 4. Valores dos odds ratios ajustados (intervalo com $95 \%$ de confiança) obtidos para a presença de hipercolesterolemia ou hipertrigliceridemia e tercis dos nutrientes.

\begin{tabular}{|c|c|c|c|}
\hline Variável1 & $\begin{array}{c}1^{\circ} \text { tercil } \\
\text { OR }(\text { IC } 95 \%)^{2}\end{array}$ & $\begin{array}{c}\text { Nutriente } \\
2^{\circ} \text { tercil } \\
\text { OR (IC95\%)2 }\end{array}$ & $\begin{array}{c}3^{\circ} \text { Tercil } \\
\text { OR }(\text { (IC } 95 \%)^{2}\end{array}$ \\
\hline \multicolumn{4}{|c|}{ Variável resposta: hipercolesterolemia } \\
\hline Calorias totais (kcal) & 1 & $0,92(0,64-1,35)$ & $0,86(0,57-1,29)$ \\
\hline Carboidratos (g) & 1 & $0,99(0,71-1,39)$ & $1,45(0,93-2,26)$ \\
\hline Proteínas (g) & 1 & $0,88(0,63-1,22)$ & $1,01(0,69-1,48)$ \\
\hline Lípides totais (g) & 1 & $1,18(0,79-1,77)$ & $1,87(1,07-3,27)$ \\
\hline Colesterol (mg) & 1 & $0,93(0,66-1,32)$ & $0,80(0,53-1,19)$ \\
\hline Ácido graxo saturado (g) & 1 & $0,86(0,60-1,23)$ & $0,64(0,40-1,01)$ \\
\hline Ácido linoléico (g) & 1 & $0,79(0,56-1,12)$ & $0,73(0,47-1,14)$ \\
\hline Ácido oléico (g) & 1 & $1,15(0,84-1,58)$ & $1,03(0,73-1,44)$ \\
\hline Ácido graxo trans (g) & 1 & $1,19(0,88-1,60)$ & $1,39(1,03-1,88)$ \\
\hline Álcool (g) & 1 & $0,89(0,60-1,32)$ & $1,18(0,84-1,64)$ \\
\hline Fibras totais $(\mathrm{g})$ & 1 & $1,49(1,05-2,12)$ & $1,16(0,86-1,56)$ \\
\hline Fibras de cereais (g) & 1 & $1,10(0,80-1,50)$ & $1,19(0,86-1,65)$ \\
\hline Fibras de vegetais $(\mathrm{g})$ & 1 & $0,88(0,64-1,19)$ & $0,95(0,70-1,30)$ \\
\hline Fibras das frutas $(\mathrm{g})$ & 1 & $0,78(0,55-1,10)$ & $0,83(0,54-1,26)$ \\
\hline \multicolumn{4}{|c|}{ Variável resposta: hipertrigliceridemia } \\
\hline Calorias totais (kcal) & 1 & $0,86(0,58-1,29)$ & $0,89(0,57-1,38)$ \\
\hline Carboidratos (g) & 1 & $1,02(0,71-1,47)$ & $1,40(0,87-2,27)$ \\
\hline Proteínas (g) & 1 & $1,20(0,84-1,70)$ & $1,10(0,74-1,63)$ \\
\hline Lípides totais (g) & 1 & $1,08(0,70-1,66)$ & $1,55(0,85-2,82)$ \\
\hline Colesterol (mg) & 1 & $0,92(0,64-1,33)$ & $0,74(0,48-1,13)$ \\
\hline Ácido graxo saturado (g) & 1 & $0,87(0,60-1,27)$ & $0,97(0,60-1,59)$ \\
\hline Ácido linoléico (g) & 1 & $1,01(0,70-1,48)$ & $0,77(0,48-1,23)$ \\
\hline Ácido oléico (g) & 1 & $0,70(0,50-0,98)$ & $0,90(0,62-1,31)$ \\
\hline Ácido graxo trans (g) & 1 & $1,26(0,91-1,74)$ & $1,09(0,80-1,49)$ \\
\hline Álcool (g) & 1 & $0,78(0,51-1,17)$ & $1,48(1,03-2,13)$ \\
\hline Fibras totais $(\mathrm{g})$ & 1 & $0,81(0,51-1,29)$ & $0,72(0,51-1,03)$ \\
\hline Fibras de cereais (g) & 1 & $0,92(0,65-1,28)$ & $0,89(0,63-1,27)$ \\
\hline Fibras de vegetais ( $\mathrm{g}$ ) & 1 & $0,72(0,52-1,00)$ & $1,10(0,79-1,55)$ \\
\hline Fibras das frutas (g) & 1 & $1,00(0,69-1,45)$ & $0,73(0,46-1,16)$ \\
\hline
\end{tabular}

1 Valores ajustados às calorias totais menos para calorias totais da dieta

2 Modelo ajustado para sexo (masculino vs. feminino), idade (anos), hábito de fumar (atual/passado vs. nunca), índice de massa corporal $\left(\mathrm{kg} / \mathrm{m}^{2}\right)$, obesidade central (sim vs. não), hipertensão arterial (sim vs. não), diabetes mellitus (sim vs. não). hipotireoidismo (sim vs. não)

\section{DISCUSSÃO}

Os principais achados deste estudo transversal foram: 1 ) a elevada prevalência de hiperlipidemia entre os nipobrasileiros $(81,5 \%)$, sendo que parcela semelhante de indivíduos apresentou, isoladamente, hipercolesterolemia ou hipertrigliceridemia (62\% e $65,6 \%$, respectivamente), 2) a confirmação da associação já bem estabelecida na literatura entre hiperlipidemia e o hipotireoidismo e 3 ) as relações entre a presença de hiperlipidemia e o consumo habitual de lípides (totais, ácido oléico, saturados e trans), carboidratos, fibras e álcool.

A comparação direta do valor da prevalência de hiperlipidemia observada nesta investigação com os de outros pesquisadores é prejudicada principalmente em função das diferenças no delineamento do estudo, na população investigada e nos critérios utilizados para o diagnóstico das hiperlipidemias, contudo pode-se constatar na literatura grande variabilidade na ocorrência dessas alterações. No Brasil, dados recentes de um estudo transversal realizado no Município de São Paulo com uma amostra aleatória de 2.103 indivíduos de 15 a 59 anos mostraram que $8,1 \%$ e $14,4 \%$ dos indivíduos adultos apresentaram, respectivamente, níveis elevados de colesterol ou de triglicérides. Esses níveis se elevavam com o aumento da idade (23). Já em Campos dos Goytacazes, no Rio de Janeiro, com a população local acima de 18 anos, 
Souza e cols. (24) observaram que 4,2\% dos indivíduos apresentavam hipercolesterolemia, 17,1\% hipertrigliceridemia e 24,2\% dislipidemia (hipercolesterolemia, hipertrigliceridemia ou níveis baixos de HDL). Washio e cols. (25), no Japão, mostraram que 23,6\% dos japoneses acima de 30 anos que foram submetidos a angiografia pela primeira vez apresentavam hipercolesterolemia e $34,7 \%$ apresentavam hipertrigliceridemia. Já López (26), em seu estudo realizado com uma amostra de mulheres de diferentes regiões da Espanha obtida por um censo demográfico e seguindo o mesmo critério de classificação usado no estudo com os nipo-brasileiros, encontrou resultados muito semelhantes: $63,1 \%$ das mulheres espanholas apresentavam hipercolesterolemia. Entre os norte-americanos, esses níveis também são elevados, onde 48 a 53\% dos americanos apresentavam colesterol elevado (27).

Neste estudo, apesar de a prevalência de hiperlipidemia observada entre os nipo-brasileiros ter sido elevada, esse achado não foi uma surpresa. Dados publicados anteriormente pelo Grupo de Estudo do Diabetes (JBDS) nessa comunidade mostraram que esses indivíduos apresentam alto risco para as doenças que compõem a síndrome metabólica, particularmente a intolerância à glicose e a hipertensão arterial, provavelmente em função de associações entre fatores genéticos e ambientais (9-12,28-31).

Com base nos fatores de risco clássicos para as doenças cardiovasculares, a idade, o sexo, o tabagismo, o sedentarismo, a hipertensão arterial sistêmica, a obesidade central, valores elevados de IMC e a intolerância a glicose $(3,20,23,26,33-37)$. No presente estudo, apenas para o sedentarismo não se observou associação estatisticamente significante com a presença de hiperlipidemia.

A relação entre as presenças de hipotireoidismo e hiperlipidemias já foi relatada por diversos pesquisadores (38-46). No presente estudo, observou-se que, do total de indivíduos com hipotireoidismo $(n=121), 89,3 \%$ apresentavam, simultaneamente, hiperlipidemia. Em nosso meio, Castro e cols. (47), em estudo realizado com uma amostra de indivíduos atendidos em ambulatório da clínica-escola de Botucatu (São Paulo), reportaram que 40 a $60 \%$ dos indivíduos com hipotireoidismo apresentavam algum distúrbio no metabolismo de lípides.

O papel da dieta no desenvolvimento de diversas doenças crônicas tem merecido grande atenção de pesquisadores em diferentes partes do mundo. Estudos com populações migrantes, geneticamente homogêneas, oferecem uma oportunidade ímpar para a realização de investigações com o objetivo de estabelecer o papel dos fatores ambientais na etiologia de doenças crônico-degenerativas (48).
A população de ancestrais japoneses é um modelo interessante, pois originariamente essa população apresentava baixa morbidade por diabetes mellitus e doenças cardiovasculares. Porém mudanças sócio-culturais, em pouco tempo, modificaram o perfil de morbi-mortalidade desses indivíduos, que passaram a apresentar alto risco para essas doenças crônico-degenerativas (49).

A dieta oriental resulta de uma cultura milenar e caracteriza-se por aspectos relacionados ao paladar e à disponibilidade de alimentos no Japão. Este inclui, entre outras características, cuidados com a saúde e a arte culinária. O estado nutricional já era fator de preocupação nos séculos passados, período em que a alimentação japonesa constituía-se de arroz que podia ser ou não misturado à cevada e ao centeio, verduras cozidas com shoyu, conservas de nabo, vegetais folhosos (tsukemono), sopa de missô e de pescados, mariscos e algas marinhas (50). A emigração proporcionou alterações no perfil nutricional e de saúde dos japoneses e as modificações nos hábitos alimentares contribuíram para o aumento do peso corporal observado em imigrantes que vivem nos Estados Unidos e no Brasil.

Costa e cols. (51), utilizando dados da primeira fase do estudo com nipo-brasileiros, observaram que a dieta dos nipo-brasileiros da cidade de Bauru era composta por $54 \%$ de carboidratos, $13,5 \%$ de proteínas e 32,5\% de lípides. Esses dados estão mais próximos dos de migrantes japoneses que vivem em Seattle, nos Estados Unidos (carboidratos: 48,5\%, proteínas: 16,5\% e lípides: $32,4 \%$ ) do que de japoneses que vivem no Japão (carboidratos: $61,1 \%$, proteínas: $14,8 \%$ e lípides: 16,7\%). Posteriormente, Freire e cols. (12), nesta mesma população, confirmaram esses valores estratificados para sexo e geração.

A mudança no hábito dietético da migração japonesa é um fator a ser levado em consideração quando se fala em prevalência de hiperlipidemia. Porém, estabelecer relação entre doença-nutriente é complexo, requerendo cautela na análise dos dados. No presente trabalho, observou-se associação entre alto consumo de ácido oléico e fibras provenientes de cereais e menores prevalências de hiperlipidemia, achados estes que concordam com a literatura.

O ácido oléico é considerado um ácido graxo monoinsaturado e muitas vezes classificado como ômega-9. É amplamente conhecido por sua ação antitrombótica, por inibir a agregação plaquetária e reduzir os níveis de colesterol total e da fração LDL, sem alterar a fração da $\operatorname{HDL}(1,3,52)$. Populações de alguns países mediterrâneos com consumo de dietas com elevadas porcentagens de gordura total (mais de $40 \%$ do total de calorias) derivadas principalmente de 
ácidos graxos monoinsaturados apresentam taxas baixas de doenças coronarianas (53)

O mecanismo responsável pela redução dos níveis sanguíneos de colesterol por meio do consumo de fibras dietéticas está relacionado à sua solubilidade e viscosidade, uma vez que esta tem a capacidade de se ligar ao ácido biliar e inibir a síntese de colesterol, provavelmente após fermentação no cólon intestinal $(54,55)$. Anderson e cols. (56), em seu estudo utilizando dieta enriquecida com farelo de aveia e feijão, observaram redução de $26 \%$ do colesterol sérico total em um período de 24 semanas de acompanhamento.

Diferentemente do esperado, observou-se que um maior teor de ácido graxo saturado na dieta associou-se a menor prevalência de hiperlipidemia, e que o maior consumo de fibras associou-se a maior prevalência de hipercolesterolemia. Estes achados não se encontram respaldados na literatura e, muito provavelmente, são decorrentes do acaso, bem como da possível mudança nos hábitos alimentares devido ao conhecimento da doença.

Estudos anteriores já reportavam que dietas ricas em ácido graxo saturado aumentavam os níveis de colesterol sérico e aumentavam a incidência de doenças cardiovasculares, em especial a aterogênese (53,57-61).

Bassett e cols. (62) compararam a dieta de japoneses e havaianos, uma vez que a prevalência de doenças cardiovasculares entre esses dois grupos era bastante distinta. Como os fatores ambientais eram semelhantes, procuraram estabelecer as relações com a dieta. Foi notado que os havaianos consumiam menos fibras e mais ácidos graxos saturados quando comprados aos japoneses.

Em estudos epidemiológicos, tanto o teor total de lípides da dieta, o consumo excessivo de ácido graxo saturado e de colesterol quanto a baixa ingestão de ácido polinsaturado estão consistentemente associados com maiores taxas de morbi-mortalidade por doenças cardiovasculares (63-65). O Seven Countries Study realizado por Keys e cols. (66), na Finlândia, Grécia, Holanda, Iugoslávia, Estados Unidos, Itália e Japão, investigou o papel da dieta nas doenças cardiovasculares e confirmaram que há associação entre a maior prevalência de doenças cardiovasculares e o consumo elevado de ácidos graxos saturados.

Atualmente, sabe-se que não apenas a quantidade total dos lípides da dieta é importante, mas é relevante estudar também a qualidade dos lípides. Os resultados de pesquisas epidemiológicas corroboraram com a hipótese de que o tipo de gordura é um determinante mais importante do risco cardiovascular que a sua quantidade total. Ensaios clínicos também mostraram que a substituição de ácido graxo saturado e gorduras trans da dieta por ácido graxo polinsa- turado é mais efetiva na redução dos níveis séricos de colesterol e da morbi-mortalidade por doenças cardiovasculares que a simples redução do teor total de lípides da dieta $(65,67-75)$. A recomendação atual é a de estimular o consumo de óleos vegetais (especialmente os de oliva e canola), de peixes e frutas oleaginosas, e reduzir o de alimentos ricos em gorduras saturadas e trans. Neste estudo, os resultados encontrados são concordantes com aqueles observados em outras pesquisas, sugerindo que o consumo reduzido de lípides totais e aumentado de ácido oléico pode ser benéfico para a redução do risco cardiovascular.

A composição dos ácidos graxos da dieta pode provocar mudanças nas concentrações plasmáticas de colesterol (76). Estudos epidemiológicos anteriores sugerem associação positiva entre consumo de ácidos graxos trans e a ocorrência de doenças cardiovasculares. Esse tipo de ácido graxo reduz os níveis de HDL e aumenta o de LDL, e já há evidências de que estes aumentam as concentrações plasmáticas de triglicérides (77-80). O estudo prospectivo Nurse's Health Study mostrou que mulheres que consumiam mais que 3\% das calorias totais diárias de ácidos graxos trans apresentavam 50\% mais chances de desenvolver alguma doença cardíaca quando comparadas àquelas que consumiam a quantidade recomendada desse tipo de gordura (até 1\% das calorias totais diárias) (81).

Sabe-se que o consumo de carboidratos está associado com os teores de triglicérides séricos (82). O indivíduo hipertrigliceridêmico deve reduzir o consumo de carboidratos simples e controlar o de carboidratos complexos $(61,83)$. É recomendado o uso de hortaliças, leguminosas, grãos integrais e frutas. Embora dietas com baixo índice glicêmico possam reduzir o perfil lipídico, a capacidade dos indivíduos para manter essas dietas em longo prazo não está bem estabelecida. Não são encontradas evidências suficientes para recomendar o uso de alimentos de baixo índice glicêmico como estratégia primária no plano alimentar (84). Neste estudo, essa relação foi confirmada entre aqueles com hipertrigliceridemia, mas sem hipotireoidismo. Situação semelhante foi também observada para o consumo de álcool. No estudo de Framingham, teve-se evidências de que o consumo moderado de álcool está relacionado com menores taxas de mortalidade por doença coronariana $(85)$. Esse efeito foi atribuído à sua capacidade de elevar a concentração da HDL e na redução do fibrinogênio $(86,87)$, enquanto que altas quantidades estão relacionadas a inúmeros efeitos adversos relacionados às doenças cardiovasculares $(82,88)$.

Uma limitação deste estudo é a sua característica transversal, o que impede o estabelecimento de inferência 
causal. A possibilidade de erro de classificação dos indivíduos quanto à presença de hiperlipidemia não pode ser descartada, mas acredita-se que esse efeito possivelmente foi minimizado pela adoção de técnicas padronizadas tanto na coleta como nos procedimentos laboratoriais. Não se pode ignorar que as associações encontradas podem refletir mudanças nos hábitos alimentares decorrentes do diagnóstico prévio de doenças. O QFCA utilizado nesse estudo foi testado e validado com a própria população do estudo, o que provavelmente minimizou a ocorrência de outros vícios de informação. Segundo Pereira \& Koifman (89), esse tipo de questionário é capaz de produzir uma classificação relativa dos indivíduos investigados com um nível aceitável de validade, sendo, portanto, de grande utilidade em estudos sobre o papel da dieta na etiologia das doenças crônicas.

Conclui-se que mudanças no estilo de vida, particularmente no hábito alimentar, podem levar a uma melhora do quadro hiperlipidêmico e que o consumo de lípides pode ser um dos principais fatores para o aumento da hiperlipidemia. Estudos prospectivos auxiliarão para testar essas hipóteses nos nipo-brasileiros de Bauru.

\section{APÊNDICE}

Alcides Hirai, MD; Amélia T Hirai, MD; Helena Harima, MD; Katsumi Osiro MD; Mário Kikuchi PhD; Sandra RG Ferreira, MD, PhD; Suely GA Gimeno, PhD (Preventive Medicine Dpt., Federal University of São Paulo, Brazil); Vania D’ Almeida, PhD (Pedriatrics Dpt., Genetics Laboratory, Federal University of São Paulo, Brazil); Laércio J Franco, MD, PhD (Social and Preventive Medicine Dpt., Medical School of Ribeirão Preto, São Paulo University, Brazil); Luiza Matsumura, $\mathrm{MD}, \mathrm{PhD}$; Regina S Moisés, MD, PhD (Internal Medicine Dpt., Federal University of São Paulo, Brazil); Marly A Cardoso, PhD (Nutrition Dpt., Faculty of Public Health, São Paulo University, Brazil); Newton de Barros Jr., MD, PhD (Surgery Dpt., Federal University of São Paulo, Brazil); Nilce Tomita, PhD (Faculty of Odontology of Bauru, São Paulo University, Brazil); Katsunori Wakisaka (Japanese-Brazilians Study Center, Brazil); and Rita Chaim (Nutrition Dpt., Sagrado Coração de Jesus University, Bauru, Brazil).

\section{AGRADECIMENTOS}

A todos os pesquisadores do Japanese Brazilians Diabetes Study, à Fundação de Amparo à Pesquisa do Estado de São Paulo - FAPESP, pelo financiamento da $2^{\text {a }}$ fase do estudo, e à Coordenação de Aperfeiçoamento de Pessoal de Nível Superior - CAPES, pela bolsa de estudo concedida.

\section{REFERÊNCIAS}

1. Costa RP, Silva CC. Doenças cardiovasculares. In: Cuppari L. Nutricão clínica do adulto. Guias de Medicina Ambulatorial e Hospitalar. UNIFESP/EPM. São Paulo: Manole, 2002.

2. Botrel TEA, Costa RD, Costa MD, Costa AMDD. Doenças cardiovasculares: causas e prevenção. Rev Bras Clin Terap 2000;26(3):87-90.

3. III Diretrizes Brasileiras sobre dislipidemias e diretriz de prevenção da aterosclerose do Departamento de Aterosclerose da Sociedade Brasileira de Cardiologia. Arq Bras Cardiol 2002.

4. NCEP. Executive summary of the Third Report of the National Cholesterol Education program (NCEP) Expert panel on detection, evaluation and treatment of high blood cholesterol in adults. JAMA 2001;285:2486-97.

5. Sociedade Brasileira de Endocrinologia e Metabologia. Distrito Federal. Projeto Conexão D: as relações entre a tireóide e a dislipidemia. Disponível em: <http:// www.ambr.com.br/sbemdf/conexaod.htm>. Acessado em fevereiro de 2005.

6. Guthrie HA, Picciano MF. Human nutrition. Missouri: Mosby, 1995.

7. Fujimoto WY, Leonetti DL, Kinyoun JL, Shuman WP, Stolov WC, Wahl PW. Prevalence of complications among secondgeneration Japanese-American men with diabetes, impaired glucose tolerance or normal glucose tolerance. Diabetes 1987;36:730-9.

8. Boyko EJ, Courten M, Zimmet PZ, Chitson P, Tuomilehto J, Albert KG. Features of the metabolic syndrome predict higher risk of diabetes and impaired glucose tolerance: a prospective study in Mauritius. Diabetes Care 2000;23:1242-8.

9. Gimeno SGA, Ferreira SRG, Franco LJ, Hirai AT, Matsumura LK, Moisés RCS. Prevalence and 7-year incidence of type 2 diabetes mellitus in a Japanese-Brazilian population: an alarming public health problem. Diabetologia 2002;45:1635-8.

10. Franco LJ. Diabetes in Japanese-Brazilians - influence of the acculturation process. Diab Res Clin Pract 1996;34:51-7.

11. Nascimento R, Franco LJ, Gimeno SGA, Hirai AT, Ferreira SRG. Diabetes mellitus tipo 2: fatores preditivos na população nipo-brasileira. Arq Bras Endocrinol Metab 2003;47(5):584-92.

12. Freire RD, Cardoso MA, Shinzato AR, Ferreira SRG. Nutritional status of Japanese-Brazilian subjects: comparison across gender and generation. Br J Nutr 2003;89:705-12.

13. Cardoso MA, Kida AA, Tomita LY, Stocco PR. Reproducibility and validity of a food frequency questionnaire among women of Japanese ancestry living in Brazil. Nutr Res $2001 ; 21: 725-33$.

14. Block G, Coyle LM, Hartman AM, Scoppa SM. Revision of dietary analysis software for the health habits and history questionnaire. Am J Epidemiol 1994;139:1190-6.

15. Fundação Instituto Brasileiro de Geografia e Estatística. Tabela de composição de alimentos. 4a ed. Rio de Janeiro: IBGE, 1996.

16. Resources Research Council, Science and Technology Agency. Standard Tables of food composition in Japan. $4^{\text {th }}$ ed. Tokyo: Women's University of Nutrition Press, 1993.

17. Willet WC. Nutritional epidemiology. $2^{\text {nd }}$ ed. New York: Oxford University Press, 1998.

18. World Health Organization (WHO). Obesity: preventing and managing the global epidemic. Report of WHO Consulation on Obesity. Geneva: 1998. 
19. Inoue $S$, Zimmet $P$. The Asian-Pacific perspective: redining obesity and its treatment. Health Communications Australia Pty limited, Sidney, Australia, 2000.

20. Chobanian AV, Bakris GL, Black HR, Cushman WC, Green LA, Izzo JL, et al; and the National High Blood Pressure Education Program Coordinating Committee. The Seventh Report of the joint national committee on prevention, detection, evaluation and treatment of high blood pressure (JNC-7). Hypertension 2003;42:1206-52.

21. Alberti KGMM, Zimmet $P Z$. Definition, diagnosis and classification of diabetes mellitus and its complications. Report of a WHO Consulation. Geneva: 1999.

22. Statacorp. Stata statistical software: release $\mathbf{8 . 0 2}$ College Station, TX Stata Corporation, 2003.

23. Centro de Vigilância Epidemiológica da Secretaria de Saúde do Estado de São Paulo (CVE). Fatores de risco para doenças crônicas. Coord: Luiz Francisco Marcopito, 2002.

24. Souza LJ, Souto Filho JTD, Souza TF, Reis AFF, Gicovate Neto C, Bastos DA, et al. Prevalência de dislipidemia e fatores de risco em Campos dos Goytacazes, RJ. Arq Bras Cardiol 2003;81(3):249-56.

25. Washio M, Sasazuki S, Kodama H, Yoshimasu K, Liu Y, Tanaka $\mathrm{K}$, et al. Role of hypertension, dyslipidemia and diabetes mellitus in the development of coronary atherosclerosis in Japan. Jap Circ J 2001;65:731-7.

26. López AMM, Miguel EM, Meabe YS, Gofinondo IN, Chivite JPSL, Gorostiza JDS, et al. Prevalencia de los principales factores de riesgo cardiovascular em mujeres de Vizcaya. Rev Esp Cardiol 2003;56(8):783-8.

27. American Heart Association. Cholesterol. Bioestatistics Fact Sheets 1998;1-3.

28. Ferreira SRG, lunes M, Franco LJ, lochida LC, Hirai A, Vivolo $M A$, et al. Disturbances of glucose and lipid metabolism in first and second generation Japanese-Brazilians. Diab Res Clin Pract 1996;34(suppl):S59-63.

29. Ferreira SRG, Franco LJ, Gimeno SGA, lochida LC, lunes M. Is insulin or its precursor independently associated with hypertension? An epidemiological study in Japanese-Brazilians. Hypertension 1997;30(part 2):641-5.

30. Lerario DDG, Gimeno SGA, Franco LJ, lunes M, Ferreira SRG e JBDS. Excesso de peso e gordura abdominal para a síndrome metabólica em nipo-brasileiros. Rev Saúde Pública 2002;36(1):4-11.

31. Sartorelli DS, Freire RD, Ferreira SRG, Cardoso MA; pelo JBDS. Dietary fiber and glucose tolerance in Japanese Brazilians. Diabetes Care 2005;28:2240-2.

32. Duncan BB, Schimidt MI, Polanczyk CA, Homrich CS, Rosa RS, Achitti AC. Fatores de risco para doenças nãotransmissíveis em área metropolitana na região sul do Brasil, prevalência e simultaneidade. Rev Saúde Pública 1993;27(1):143-8.

33. Valença MM. Dislipidemia e outros fatores de risco no acidente vascular cerebral isquêmico. An Fac Med Univ Fed Pernamb 1994;39(2):96-105.

34. Fernandes IC, Sá SV, Silva AMSP, Curi AN, Freire CRS, Almeida ML, et al. Diabetes "mellitus" e dislipidemia. JBM 2000;79(3):39-44.

35. Dudeja V, Misra A, Pandey RM, Devina G, Kumar G, Vikram NK. BMI does not accurately predict overweight in Asian Indians in northern India. Br J Nutr 2001;86:105-12.

36. Amato RV, César LAM, Mansur AP, Hueb WA, Martins JRM, Vianna CB, et al. Manifestação clínica da doença coronariana e fatores de risco em imigrantes japoneses e seus descendentes em São Paulo. Arq Bras Cardiol 2003;81(3):229-33.

37. Dias INB, Galil AGS, Sousa SMC, Rocha LM, Ferreira MA, Simas ECVS, et al. Análise de fatores de risco cardiovasculares e complicações de hipertensos e diabéticos tabagistas num serviço do SUS. Diab Clin 2004;5:366-70.

38. Valdemarsson S, Hansson P, Hedner P, Nilsson-Ehle P. Relations between thyroid function, hepatic and lipoprotein lipase activities and plasma lipoprotein concentrations. Acta Endocrinol 1983;104(1):50-6.
39. O'Brien T, Dinneen SF, O’Brien PC, Palumbo PJ. Hiperlipidemia in patients with primary and secondary hypothyroidism. Mayo Clin Proc 1993;68(9):860-6.

40. Diekman T, Lansberg PJ, Kastelein JJ, Wiersinga WM. Prevalence and correction of hypothyroidism in a large cohort of patients referred for dyslipidemia. Arch Intern Med 1995; 155(14):1490-5.

41. Canaris GJ, Manowitz NR, Mayor G, Ridgway EC. The Colorado thyroid disease prevalence study. Arch Intern Med 2000;160(4):526-34.

42. Efstathiadou Z, Bitsis S, Milionis HJ, Kukuvitis A, Bairaktari $E T$, Elisaf MS, et al. Lipid profile in subclinical hypothyroidism: is L-thyroxine substitution beneficial? Eur $\mathbf{J}$ Endocrinol 2001;145(6):705-10.

43. Duntas LH. Thyroid disease and lipids. Thyroid 2002;12(4):287-93.

44. Al-Tonsi AA, Abdel-Gayoum AA, Saad M. The secondary dislipidemia and deranged serum phosphate concentration in thyroid disorders. Exp Mol Pathol 2004;76(2):182-7.

45. Hueston WJ, Pearson WS. Subclinical hypothyroidism and the risk of hypercholesterolemia. Ann Fam Med 2004;2(4):351-7.

46. Caraccio N, Dardano A, Monzani F. Subclinical thyroid disease and cardiovascular disease. JAMA 2005;293(9):1059-60.

47. Castro AVB, Bononi AP, Aragon F, Padovani CR, Nogueira CR, Mazeto GMFS, et al. Clinical and laboratory evaluation of hyperlipidemic and hypothyroid patients. Arq Bras Cardiol $2001 ; 76(2): 123-6$

48. Centro de Estudos Nipo-Brasileiros. Pesquisa da população descendente de japoneses no Brasil. São Paulo: Centro de Estudos Nipo-Brasileiros, 1998.

49. Fujimoto WY, Newel-Morris L, Grote M, Bergstrom RW, Shuman WP. Visceral fat and morbidity: NIDDM and atherogenic risk in Japanese men and women. Int J Obes 1991;15:41-4.

50. Japanese-Brazilian Diabetes Study Group (JBDS). Diabetes mellitus e doenças associadas em nipo-brasileiros. São Paulo: Forest, 2004. p. 133.

51. Costa MB, Ferreira SRG, Franco LJ, Gimeno SGA, lunes M and JBDS. Dietary patterns in a high-risk population for glucose intolerance. J Epidemiol 2000;10(2):111-7.

52. Schaefer EJ. Lipoproteins, nutrition, and heart disease. Am J Clin Nutr 2002;75:191-212.

53. Ascherio A, Willett W. New directions in dietary studies of coronary heart disease. J Nutr 1995;12S(suppl. 3):647S-55.

54. American Dietetic Association (ADA). Position of ADA: Health implications of dietary fiber. J Am Diet Assoc 2002;102(7):993-1000.

55. Wu H, Dwyer KM, Fan Z, Shicore A, Fan J, Dwyer JH. Dietary fiber and progression of atherosclerosis: The Los Angeles Atherosclerosis Study. J Nutr 2003;78:1085-91.

56. Anderson JW, Smith BM, Gustafson NJ. Health benefits and practical aspects of high-fiber diets. Am $\mathbf{J}$ Clin Med 1994;59(suppl):1242S-7.

57. Ahrens EM, Hirsch J, Insull W, Tsaltas TT, Blomstrand R, Peterson ML. The influence of dietary fats on serum lipids in man. Lancet 1957;272:943-53.

58. Grundy SM, Bonanome A. Workshop on monounsaturated fatty acids. Arteriosclerosis 1987;7(6):644-8.

59. Katan MB, Zock PL, Mensink RP. Dietary oils, serum lipoproteins and coronary heart disease. Am $J$ Clin Nut 1994;61(suppl):1368S-73.

60. Metz DA, Kris-Etherton PM, Morris CD, Mustad VA, Stern JS, Oprail S, et al. Dietary compliance and cardiovascular risk reduction with prepared meal plan compared with a selfselected diet. Am J Clin Nutr 1997;66:373-85.

61. Alves DC, Gerude M. Dislipidemias - Distúrbio do metabolismo dos lipídios. In: Augusto ALP, Alves DC, Mannarino IC, Gerude M. Terapia nutricional. São Paulo: Atheneu, 1999. pp. 207-14.

62. Bassett DR, Abel M, Moellering Jr RC, Rosenblatt G, Strokes $\mathrm{J}$. Coronary heart disease in Hawaiian dietary intake, depot fat, "stress", smoking and energy balance in Hawaiian and Japanese men. Am J Clin Nutr 1969;22(11):1483-503. 
63. Tell GS, Evans GW, Folsom AR, Shimakawa T, Carpenter MA, Heiss G. Dietary fat intake and carotid artery wall thickness. The Atherosclerosis Risk in Communities (ARIC) Study. Am J Epidemiol 1994;139:979-89.

64. Morris MC, Manson JE, Rosmer B, Buring JE, Willet WC, Hennekens $\mathrm{CH}$. Fish consumption and cardiovascular disease in the Physicians' Health Study: a prospective study. Am J Epidemiol 1995; 142:166-75.

65. Albert CM, Hennekens $\mathrm{CH}$, O'Donnell C, Ajani UA, Willett WC, Ruskin JN, et al. Fish consumption and risk of sudden cardiac death. JAMA 1998;279:23-9.

66. Keys A. The Seven Countries Study. Circulation 1970;41(suppl):162S-98.

67. Ascherio A, Rimm EB, Giovannucci EL, Spielgelman D, Stampfer M, Willett WC. Dietary fat and risk of coronary heart disease in men: cohort follow-up study in the United States. BMJ 1996;313:84-90.

68. Appel LJ, Moore TJ, Obarzanek E, Vollmer WM, Svetkey LP, Sack FM, et al; DASH Collaborative Research Group. A clinical trial of the effect of dietary patterns on blood. $\mathbf{N}$ Engl $\mathbf{J}$ Med 1997;336:1117-24.

69. Hu FB, Manson JE, Stampfer M, Colditz G, Liu S, Salomon CG. Diet, lifestyle, and risk of type 2 diabetes in women. $\mathbf{N}$ Engl J Med 2001;345:790-7.

70. Hu FB, Manson JE, Willett WC. Types of dietary fat and risk of coronary heart disease: a critical review. J Am Coll Nutr 2001;20:5-19.

71. Liu S, Lee IM, Ajani U, Cole SR, Buring JE, Manson JA. Intake of vegetables rich in carotenoids and risk of coronary heart disease in men: The Physicians' Health Study. Int J Epidemiol 2001;30:130-5.

72. Boniface DR, Tefft ME. Dietary fats and 16-year coronary heart disease mortality in a cohort of men and women in Great Britain. Eur J Clin Nutr 2002;56:786-92.

73. He K, Merchant A, Rimm EB, Rosner BA, Stampfer MJ, Willett WC. Dietary fat intake and risk of stroke in male US healthcare professional: 14-year prospective cohort study. BMJ 2003;327:1-6

74. Jakobsen UM, Overvad K, Dyerberg J, Schroll M, Heitmann BL. Dietary fat and risk of coronary disease: possible effect modification by gender and age. Am J Epidemiol 2004;160:141-9.

75. Rastogi T, Reddy KS, Vaz M, Spiegelman D, Prabhakaran D Willett WC, et al. Diet and risk of ischemic heart disease in India. Am J Clin Nutr 2004;79:582-90.

76. Garcia RWD, Sperandio T, Padezzi J, Lopes G, Alves FR, Duarte TO. Relação entre consumo alimentar, atividade física e características antropométricas e os lipídios séricos em pacientes com dislipidemia. Rev Bras Nutr Clin 2002;17(3):99-106.

77. Hu FB, Stampfer MJ, Manson JE, Rimm E, Colditz GA, Rosner $B A$, et al. Dietary fat intake and the risk of coronary heart disease in women. N Engl J Med 1997;337:1491-9.
78. Hu FB, Stampfer MJ, Rimm E, Ascherio A, Rosner BA, Spiegelman D, et al. Dietary fat and coronary heart disease: a comparison of approaches for adjusting for total energy intake and modeling repeated dietary measurements. Am J Epidemiol 1999;149:531-40.

79. Willett WC, Stampfer MJ, Manson JE, Colditz GA, Speizer FE, Rosner BA, et al. Intake of trans fatty acids and risk of coronary heart disease among women. Lancet 1993;341:581-5.

80. Willett WC. Surprising news about fat. In: Willett WC, ed. Eat, drink and be healthy: the Harvard Medical School guide to healthy eating. New York: Simon \& Schuster Adult Publishing Group, 2001. pp. 56-84.

81. World Health Organization / Food and Agricultural Organization. Diet, nutrition and the prevention of chronic disease. Geneva: World Health Organization; 2003 (WHO Technical Report Series 916).

82. Martinez TLR. Condutas clínicas nas dislipidemia. Belo Horizonte: Health, 1997. p. 291.

83. Mahan LK, Escott-Stump S. Krause: Alimentos, nutrição \& dietoterapia. 9ạ ed. São Paulo: Roca, 1998.

84. Sociedade Brasileira de Hipertensão / Sociedade Brasileira de Cardiologia / Sociedade Brasileira de Endocrinologia e Metabologia, et al. I Diretriz Brasileira de Diagnóstico e Tratamento da Síndrome Metabólica. Arq Bras Cardiol 2005;84(suppl. 1):3-28.

85. Pearson TA. Alcohol and heart disease. Circulation 1996;94(11):3023-5.

86. Key TJ, Appleby PN. Vegetarianism, coronary risk factors and coronary heart disease. In: Sabaté J, ed. Vegetarian nutrition. USA: CRC Press, 2001. pp. 33-54.

87. Kotchen TA, Kotchen MJ. Nutrition, diet and hypertension. In: Schils ME, ed. Modern nutrition in health and disease. 9th ed. USA: Williams \& Wilkins, 1998. pp. 1217-27.

88. American Heart Association (AHA). Wine and your heart. A science advisory for healthcare professionals from the nutrition committee, council on cardiovascular nursing of The American Heart Association. Circulation 2001;103(3):472-5.

89. Pereira RA, Koifamn S. Uso do questionário de freqüência na avaliação do consumo alimentar progresso. Rev Saúde Pública 1999;33(6):610-21.

Endereço para correspondência:

Marselle Rodrigues Bevilacqua

Rua Santo Irineu 571, apto. 113

04127-120 São Paulo, SP

E-mail: ma_bevilacqua@yahoo.com.br 